\title{
Genetic variations in PRKAA1 predict the risk and progression of gastric Cancer
}

Minbin Chen ${ }^{1 \dagger}$, Baohu Jiang ${ }^{2 \dagger}$, Bangshun He ${ }^{3} \mathbb{C}$, Min Tang ${ }^{1}$, Ping Wang ${ }^{4}$, Li Chen ${ }^{5}$, Jianwei Lu ${ }^{6}$ and Peihua Lu*

\begin{abstract}
Background: PRKAA1 encodes a-subunit of 5-AMP-activated protein kinase (AMPK), which has been implicated in the pathogenesis of carcinoma of the stomach. Previous works have suggested that polymorphisms in the PRKAA1 may be associated with the risk of non-cardiac gastric cancer (NCGC), but whether PRKAA1 polymorphisms are related to clinical pathologic characteristics of gastric cancer and its clinical outcome is largely unknown.

Methods: We carried out a case-control study including a total of 481 gastric cancer patients and 490 healthy controls. The genotypes of enrolled polymorphisms were identified with Sequenom MassARRAY platform.

Results: This study showed that rs10074991 GG genotype (adjusted OR=1.44, 95\%Cl:0.99-2.09, $p=0.056$ ) has a borderline significantly increased risk for gastric cancer, which was consistent with the result of additive model (adjusted $\mathrm{OR}=1.21,95 \% \mathrm{Cl}: 1.01-1.46, p=0.042$ ). In similar, an increased risk of gastric cancer was also observed for rs13361707 TC genotype (adjusted $\mathrm{OR}=1.47,95 \% \mathrm{Cl}: 1.01-2.14, p=0.043$; additive model: adjusted $\mathrm{OR}=1.22,95 \% \mathrm{Cl}$ : $1.02-1.47, p=0.033$ ). Furthermore, the rs 154268 and rs 461404 were also found associated with increased gastric cancer risk, which may be influenced by age, tumor type and differentiation, and tumor stage. Haplotype analysis indicated A-G-C-T-C-G haplotype (rs6882903, rs10074991, rs13361707, rs3805490, rs154268 and rs461404) is associated with increased risk of gastric cancer $(\mathrm{OR}=1.29,95 \% \mathrm{Cl}: 1.02-1.62, p=0.035)$. The univariate analysis for overall survival (OS) revealed that both of rs10074991 and rs13361707 variants are associated with poor OS in patients with NCGC.

Conclusion: This case-control study provided the evidence thatrs13361707CC, rs10074991GG, rs461404GG, and rs154268CC are associated with increased gastric cancer risk, especially for NCGC, and that patients with rs10074991 G or rs13361707 C allele have a poor OS.
\end{abstract}

Keywords: Gastric cancer, Polymorphism, PRKAA1, Prognosis

\section{Background}

Gastric cancer (GC) is one of the most common cancers worldwide and remains the leading cause of cancer related death [1]. The incidence of this disease varies with the geographical region and patient ethnicity. About $70 \%$ cases in the world were reported from developing countries, and Eastern Asian countries have the highest GC incidence and mortality $[1,2]$. Although mechanism of gastric carcinogenesis is still not fully understood, environmental factors, such as high intake of salt,

\footnotetext{
* Correspondence: Iphty1_1@163.com

${ }^{\dagger}$ Minbin Chen and Baohu Jiang contributed equally to this work.

${ }^{7}$ Department of Medical Oncology, Wuxi People's Hospital of Nanjing

Medical University, No. 299, Qingyang Road, Wuxi 214023, Jiangsu Province,

China

Full list of author information is available at the end of the article
}

tobacco smoking, and particularly Helicobacter pylori $(H$. pylori) infection have been regarded as the risk factors for the disease [3].Genetic factors have also been found to contribute to the risk of $\mathrm{GC}$, with the first-degree relatives of the GC patients tending to have about 1.3 to 3.0 fold higher relative risk for GC than those without relatives with GC [4].

To date, genetic variations have widely been shown to be associated with GC risk [5], with particular importance on the polymorphisms involved in the signal transduction pathways [6, 7]. The 5-AMP-activated protein kinase (AMPK) pathway has been implicated in a series of tumors including GC. This is a heterotrimeric protein that consists of an $\alpha$-catalytic subunit and 2 regulatory subunits $(\beta$ and $\gamma)$, and the $\alpha$-subunit is encoded either

(c) The Author(s). 2018 Open Access This article is distributed under the terms of the Creative Commons Attribution 4.0 International License (http://creativecommons.org/licenses/by/4.0/), which permits unrestricted use, distribution, and 
by PRKAA1 or PRKAA2 gene. Previous genome-wide association study (GWAS) identified the PRKAA1 polymorphism rs13361707 as a risk factor for non-cardiac GC (NCGC) in a Chinese population [8]; however, these results were not successfully duplicated [9], which may be due to the different characteristics of enrolled participants, population stratification, and clinical pathologic characteristics of GC. It is also not known whether the polymorphisms in the PRKAA1 gene are related to clinical pathological characteristics of GC and clinical outcome of the patients. We carried out this case-control study on a Chinese population to investigate the susceptibility of six polymorphisms in the PRKAA1 gene (see Additional file 1.) to the risk of GC and their associations with the clinical pathological characteristics, and evaluated the predictive value of these polymorphisms to the clinical outcome of GC patients.

\section{Methods}

\section{Study subjects}

A total of 481 GC patients, and age- and gendermatched 490 healthy individuals were enrolled in this study. The patients were histologically diagnosed as GC from Nanjing First Hospital, Nanjing Medical University, and the healthy controls were individuals who came to the hospital for routine physical examinations and were confirmed be healthy. All the participants were the heritably unrelated ethnic Han Chinese from the same geographic region of Nanjing City, Jiangsu, China. The whole blood of all enrolled participants were collected before operation and then stored at $-80{ }^{\circ} \mathrm{C}$ before genotyping. The clinical features of patients, including tumor size, distant metastasis, and depth of invasion, were collected from the patients' medical records provided by Department of pathology, and the tumor TNM stages were examined and evaluated using the TNM classification according to American Joint Commission for Cancer Staging in 2002, sixth edition. The clinical outcomes of patients were found through on-site interview, direct calling, or medical chart review.

The characteristics of healthy controls, including age, gender, smoking and drinking, were collected via a questionnaire. Individuals who had smoked daily for more than 1 year were considered smokers, and those who consumed one or more alcoholic drinks per week for at least one year were considered drinkers. The protocol of this study was in accordance with the Declaration of Helsinkiand approved by the Institutional Review Board of the Nanjing First Hospital, and written informed consent was obtained from all the participants.

\section{DNA extraction and genotyping}

The genotypes of all polymorphisms were detected with the SequenomMassARRAY platform, as previously described [10, 11]. First, DNA was extracted from whole-blood samples and concentrated by using GoldMag-Mini Whole Blood Genomic DNA Purification Kit according to the manufacture's protocol (GoldMag Co. Ltd. Xi'an, China), and then DNA purity was measured by spectrometry (DU530 UV/VIS spectrophotometer, Beckman Instruments, Fullerton, CA, US). The qualified DNA samples were genotyped using the SequenomMassARRAY platform followed the standard protocol recommended by the manufacturer of a Sequenom Mass-ARRAY ${ }^{\circ}$ R1000(Sequenom, Inc.). Multiplexed SNP MassEXTENDED assay was designed by SequenomMassARRAY Assay Design 3.0 Software [12]. Finally, data management and analysis were performed using SequenomTyper 4.0 Software [12, 13].

\section{H. pylori infection detection}

H. pylori infection status of enrolled participants has been determined by serology using a commercial $H$. pylori Immunogold Testing Kit (KangmeiTianhong Biotech (Beijing) Co., Ltd., Beijing, China), which has been validated in the Chinese population with sensitivity of $98.29 \%$ and specificity of $98.51 \%$ for the detection of $H$. pylori infection.

\section{Statistical analysis}

The Hardy-Weinberg equilibrium in the healthy control group was tested by using a goodness of fit chi-square test. The statistical analysis for genotype distribution was performed by the $\mathrm{X}^{2}$ test, and odds ratios (OR) and 95\% confidence intervals (CIs) were calculated using logistic regression model. The dominant model, co-dominant model, and additive model were the test for all polymorphisms, with the dominant and co-dominant models being used only if the additive model is significant or there is a previous hypothesis to do this.

Survival curves were analyzed by the Kaplan-Meier method, and the Hazard ration (HR) and 95\% CIs were calculated using Cox proportional hazards regression model. The $P$ value $<0.05$ was considered statistically significant difference. The haplotype analysis was performed using online software SHEsis (analysis.bio-x.cn/ myAnalysis.php).

\section{Results}

\section{Characteristics of the participants}

There was no significant difference in age (cases: 65.55 \pm 11.92 years, healthy controls: $64.85 \pm 11.83 ; p=0.694$ ), gender (cases: male73.60\%, healthy controls: male73.27\%; $p=0.782$ ), smoking (cases: $23.08 \%$, healthy controls: $24.29 \% ; p=0.658$ ), and drinking (cases: $11.02 \%$, healthy controls: $9.59 \% ; p=0.465$ ) between cases and controls. For $H$. pylori infection status, the ratio of $H$. pylori infection in cases (54.47\%) was higher than that in 
healthy controls $(49.18 \%)$, however there was no significant difference between the two groups $(p=0.099)$, as presented in Table 1.

For the clinical pathological characteristics, a total of 195 (40.54\%) and 286 (59.46\%) patients had low and median to high pathological differentiation, respectively. For the tumor site classification, a total of $159(33.06 \%)$ and $322(66.94 \%)$ patients were classified to TNM stage T1-T2 and T3-T4, respectively. For the tumor location, a total of $140(29.11 \%)$ and $341(70.89 \%)$ patients were diagnosed as gastric cardiac adenocarcinoma (GCA) and non-cardiatic GC (NCGC), respectively.

\section{Association between polymorphisms and risk of GC}

The genotype distributions of the selected polymorphisms in cases and controls are presented in Table 2. The observed frequencies of all tested genotypes in controls did not deviate from Hardy-Weinberg equilibrium

Table 1 Clinical characteristics of the participants

\begin{tabular}{|c|c|c|c|}
\hline Variables & Cases, n (\%) & Controls, n (\%) & $p$-Value \\
\hline Total & 481 & 490 & \\
\hline Age (mean $\pm S D)$ & $65.55 \pm 11.92$ & $64.85 \pm 11.83$ & $0.694^{\mathrm{a}}$ \\
\hline$>60$ & 168 & 167 & $0.782^{b}$ \\
\hline$\leq 60$ & 313 & 323 & \\
\hline \multicolumn{4}{|l|}{ Gender } \\
\hline Male & $354(73.60)$ & $359(73.27)$ & $0.907^{b}$ \\
\hline Female & $127(26.40)$ & $131(26.73)$ & \\
\hline \multicolumn{4}{|l|}{ Drinking } \\
\hline Yes & $53(11.02)$ & $47(9.59)$ & $0.465^{b}$ \\
\hline No & 428(88.98) & $443(90.41)$ & \\
\hline \multicolumn{4}{|l|}{ Smoking } \\
\hline Yes & $111(23.08)$ & $119(24.29)$ & $0.658^{b}$ \\
\hline No & $370(76.92)$ & $371(75.71)$ & \\
\hline \multicolumn{4}{|c|}{ Helicobacter pylori infection status } \\
\hline Positive & $262(54.47)$ & $241(49.18)$ & $0.099^{b}$ \\
\hline Negative & $219(45.53)$ & $249(50.81)$ & \\
\hline \multicolumn{4}{|l|}{ Differentiation } \\
\hline Low & 195(40.54) & & \\
\hline Med and high & $286(59,46)$ & & \\
\hline \multicolumn{4}{|l|}{ Clinical stages } \\
\hline $\mathrm{T} 1-\mathrm{T} 2$ & 159(33.06) & & \\
\hline T3-T4 & $322(66.94)$ & & \\
\hline \multicolumn{4}{|l|}{ Tumor Site } \\
\hline GCA & $140(29.11)$ & & \\
\hline NGCA & $341(70.89)$ & & \\
\hline
\end{tabular}

GCA gastric cardiac adenocarcinoma, NGCA non-gastric

cardiac adenocarcinoma

${ }^{\mathrm{a}}$ Independent $t$-test. ${ }^{\mathrm{b}}$ Two-sided $\mathrm{x} 2$ test for distributions between cases and controls
(HWE) (rs10074991: $p=0.129 ;$ rs13361707: $p=0.152$; rs1044129: $p=0.368 ; \operatorname{rs154268:~} p=0.140 ;$ rs6882903: $p=$ 0.842; rs3805490: $p=0.929$; rs461404: $p=0.155$ ).

Rs10074991 GG genotype had a borderline significantly increased risk of GC (adjusted OR $=1.44,95 \% \mathrm{CI}$ : 0.99-2.09, $p=0.056$ ), and the additive model shows rs10074991 is an increased risk factor for GC (adjusted $\mathrm{OR}=1.21,95 \% \mathrm{CI}: 1.01-1.46, p=0.042$ ). In similar, an increased risk of rs13361707 was also observed for GC (TC vs. GG: adjusted $\mathrm{OR}=1.47,95 \% \mathrm{CI}: 1.01-2.14, p=$ 0.043; additive model: adjusted $\mathrm{OR}=1.22$, $95 \% \mathrm{CI}: 1.02$ 1.47, $p=0.033$ ). Besides, the results have also revealed that rs154268 and rs461404 are associated with increased GC risk (rs154268 TC: adjusted OR $=1.96$, 95\%CI: 1.06-3.63, $p=0.033$; rs154268 additive model: adjusted $\quad \mathrm{OR}=1.24, \quad 95 \% \mathrm{CI}: \quad 1.00-1.53, \quad p=0.053$; rs461404 GA: adjusted OR $=2.05,95 \% \mathrm{CI}: 1.11-3.78, p=$ 0.022 ; rs461404 additive model: adjusted $\mathrm{OR}=1.26$, 95\%CI: $1.01-1.56, p=0.037)$. However, there was no significant association between rs6882903 and rs3805490 and risk of GC, as summarized in Table 2.

\section{Stratification analysis}

To further assess the four potential susceptible polymorphisms (rs10074991, rs13361707, rs154268 and rs461404) to the risk of GC, a stratified analysis was performed by subgroups of participants' clinical characteristics (age, gender, H. pylori infection status), and tumor pathological characteristics (tumor site, tumor differentiation, and clinical stage).

In China, men usually retire at age of 60 , which means they retain a stable and sustainable life style (the environmental factors), so we choose 60 years as the cut-off value for the subgroup analysis. In the subgroup of age $\leq$ 60, rs10074991GG (adjusted OR $=1.93$, 95\%CI: 1.00 3.73, $p=0.050$ ), rs13361707CC (adjusted $\mathrm{OR}=2.00$, 95\%CI: 1.04-3.84, $p=0.039$ ) and rs461404GG (adjusted $\mathrm{OR}=3.12$, 95\%CI: $1.05-9.28, p=0.040)$ were associated with increased GC risk. However, in the group of age $>60$, there was no significant association of these four polymorphisms with the risk of GC. For the subgroup of gender, in the male group, rs10074991 (additive model: adjusted $\mathrm{OR}=1.25,95 \% \mathrm{CI}: 1.01-1.56, p=0.046)$ and rs13361707 (CC: adjusted OR $=1.44,95 \%$ CI: $1.01-2.06$, $p=0.044$; additive model: adjusted $\mathrm{OR}=1.27,95 \% \mathrm{CI}$ : $1.02-1.58, p=0.034$ ) contributed to increased risk of GC. In similar, in the subgroup of positive $H$. pylori infection, a borderline significantly increased risk of rs10074991 (AG: adjusted OR $=1.68$, 95\%CI: 0.98 $2.88, p=0.060$; additive model: adjusted $\mathrm{OR}=1.30$, 95\%CI: $0.99-1.69, p=0.057$ ) and rs13361707 (TC: adjusted $\mathrm{OR}=1.75,95 \% \mathrm{CI}$ : 1.02-3.00, $p=0.042$; additive model: adjusted $\mathrm{OR}=1.32,95 \% \mathrm{CI}: 1.01-1.73, p=0.041$ ) was observed for GC, as shown in Table 3. For the 
Table 2 Distribution of the genotypes in all participants

\begin{tabular}{|c|c|c|c|c|}
\hline Genotype & Controls, n (\%) & Patients, n (\%) & OR $(95 \% \mathrm{Cl})^{\mathrm{a}}$ & $p$-Value \\
\hline \multicolumn{5}{|l|}{ rs10074991 } \\
\hline AA & $128(26.12)$ & $104(21.62)$ & Reference & \\
\hline$A G$ & $261(53.27)$ & 255(53.01) & $1.19(0.87,1.62)$ & 0.283 \\
\hline GG & $101(20.61)$ & $122(25.36)$ & 1.44(0.99,2.09) & 0.056 \\
\hline$A G / G G$ & $362(73.88)$ & 377(78.38) & $1.26(0.94,1.70)$ & 0.127 \\
\hline Additive model & & & $1.21(1.01,1.46)$ & 0.042 \\
\hline \multicolumn{5}{|l|}{ rs13361707 } \\
\hline$\pi$ & $129(26.33)$ & 103(21.41) & Reference & \\
\hline $\mathrm{TC}$ & 260(53.06) & $256(53.22)$ & $1.22(0.89,1.66)$ & 0.219 \\
\hline CC & $101(20.61)$ & $122(25.36)$ & $1.47(1.01,2.14)$ & 0.043 \\
\hline $\mathrm{TC} / \mathrm{CC}$ & $361(73.67)$ & $365(75.88)$ & $1.29(0.96,1.74)$ & 0.093 \\
\hline Addictive model & & & $1.22(1.02,1.47)$ & 0.033 \\
\hline \multicolumn{5}{|l|}{ rs154268 } \\
\hline$\pi$ & $297(60.61)$ & 271(56.34) & Reference & \\
\hline $\mathrm{TC}$ & $176(35.92)$ & 179(37.21) & $1.13(0.86,1.47)$ & 0.388 \\
\hline CC & 17(3.47) & $31(6.44)$ & $1.96(1.06,3.63)$ & 0.033 \\
\hline $\mathrm{TC} / \mathrm{CC}$ & 193(39.39) & $210(43.66)$ & $1.20(0.93,1.56)$ & 0.158 \\
\hline Additive model & & & $1.24(1.00,1.53)$ & 0.053 \\
\hline \multicolumn{5}{|l|}{ rs6882903 } \\
\hline CC & $342(69.80)$ & $312(64.86)$ & Reference & \\
\hline CA & 134(27.35) & 149(30.98) & $0.86(0.41,1.80)$ & 0.687 \\
\hline $\mathrm{AA}$ & $14(2.86)$ & $20(4.16)$ & $1.56(0.77,3.15)$ & 0.217 \\
\hline CA/AA & 148(30.20) & 169(35.14) & $1.26(0.96,1.65)$ & 0.097 \\
\hline Additive model & & & $1.23(0.98,1.55)$ & 0.078 \\
\hline \multicolumn{5}{|l|}{ rs3805490 } \\
\hline$\pi$ & 279(56.94) & $280(58.21)$ & Reference & \\
\hline $\mathrm{TA}$ & $181(36.94)$ & 170(35.34) & $0.93(0.71,1.21)$ & 0.567 \\
\hline AA & $30(6.12)$ & $31(6.44)$ & $1.02(0.60,1.73)$ & 0.953 \\
\hline TA/AA & $211(43.06)$ & 201(41.79) & $0.94(0.73,1.21)$ & 0.627 \\
\hline Additive model & & & $0.97(0.79,1.19)$ & 0.756 \\
\hline \multicolumn{5}{|l|}{ rs461404 } \\
\hline AA & 298(60.82) & $270(56.13)$ & Reference & \\
\hline GA & 175(35.71) & 179(37.21) & $1.14(0.87,1.49)$ & 0.341 \\
\hline GG & $17(3.47)$ & $32(6.65)$ & $2.05(1.11,3.78)$ & 0.022 \\
\hline GA/GG & 192(39.18) & $211(43.87)$ & $1.22(0.95,1.58)$ & 0.125 \\
\hline Additive model & & & $1.26(1.01,1.56)$ & 0.037 \\
\hline
\end{tabular}

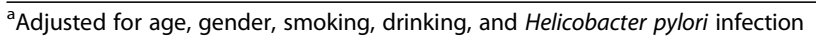

subgroup of pathological characteristics of tumor, the four polymorphisms were significant associated with increased risk of NCGC, but not GCA. Moreover, the significant associations of these four polymorphisms were observed in the subgroup of patients with tumor in median or high differentiation or T3-T4, but not for low differentiation or T1-T2, as shown in Table 4.

\section{Haplotype analysis of polymorphisms in PRKAA1}

The enrolled six polymorphisms locate in the intron or upstream of PRKAA1, so these sites may be in linkage disequilibrium with each other. Therefore, the combined susceptibility of these six polymorphisms to GC risk was calculated by haplotype analysis. The results indicated that the haplotype A-G-C-T-C-G (rs6882903, 


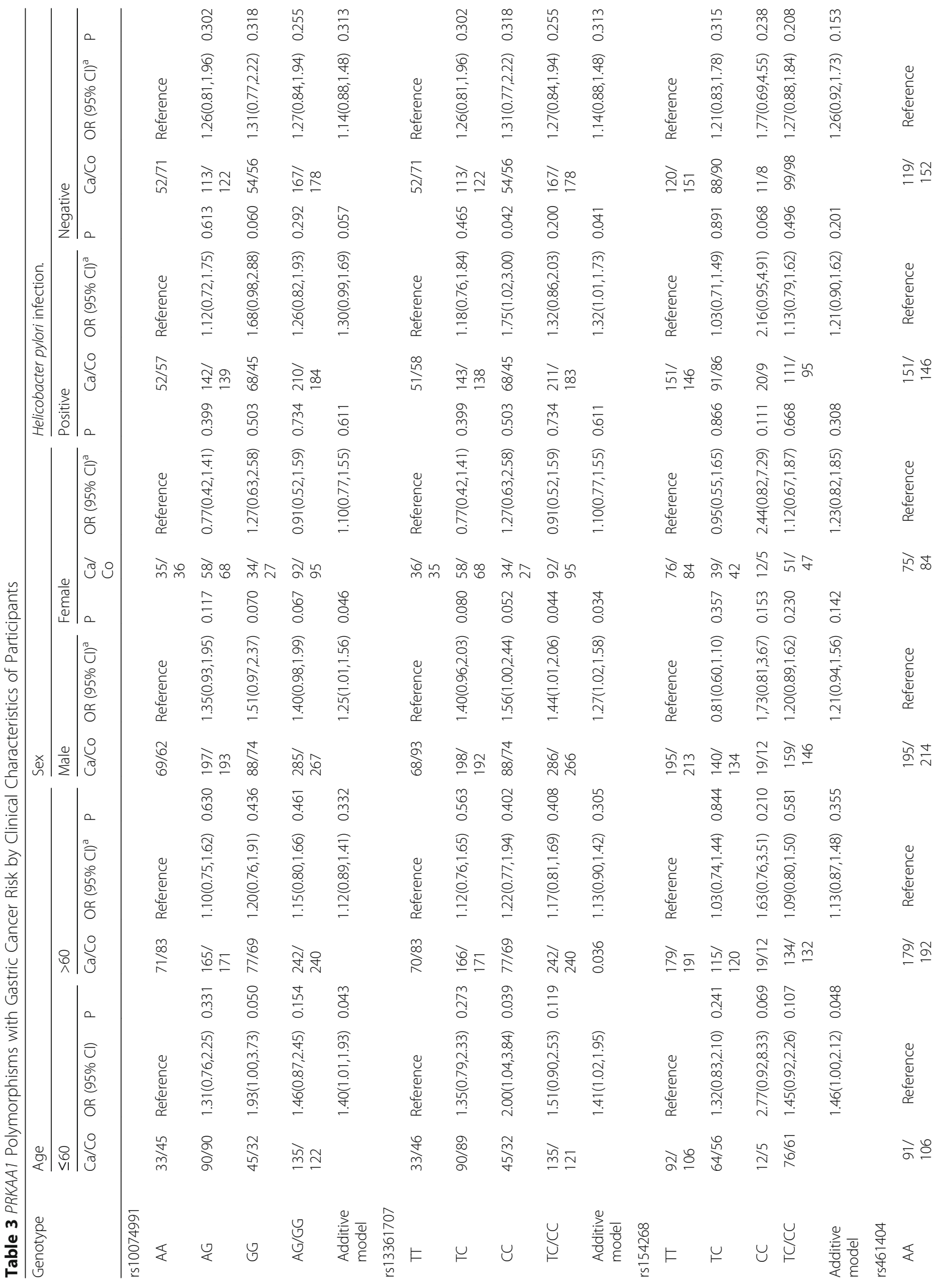




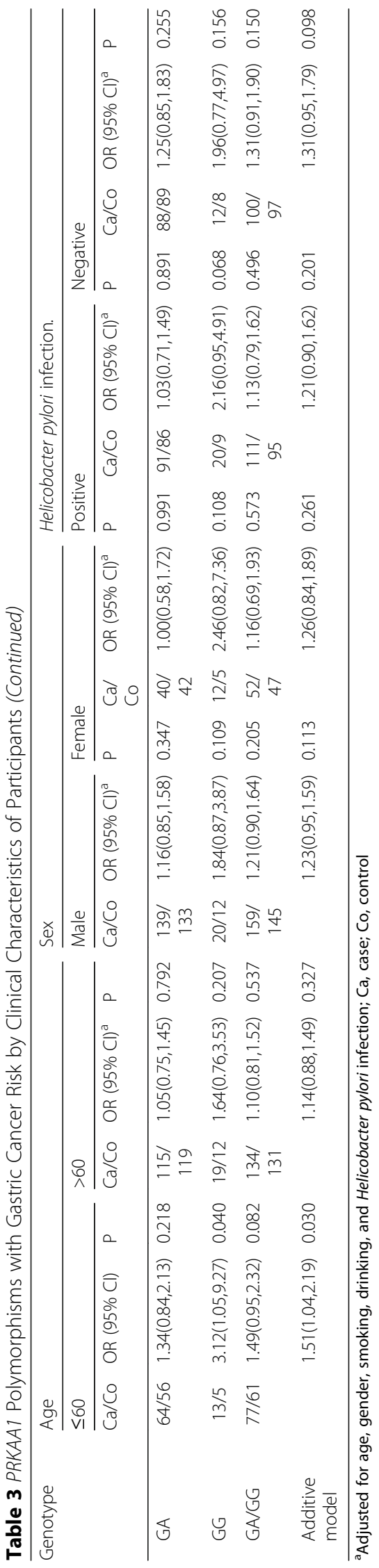




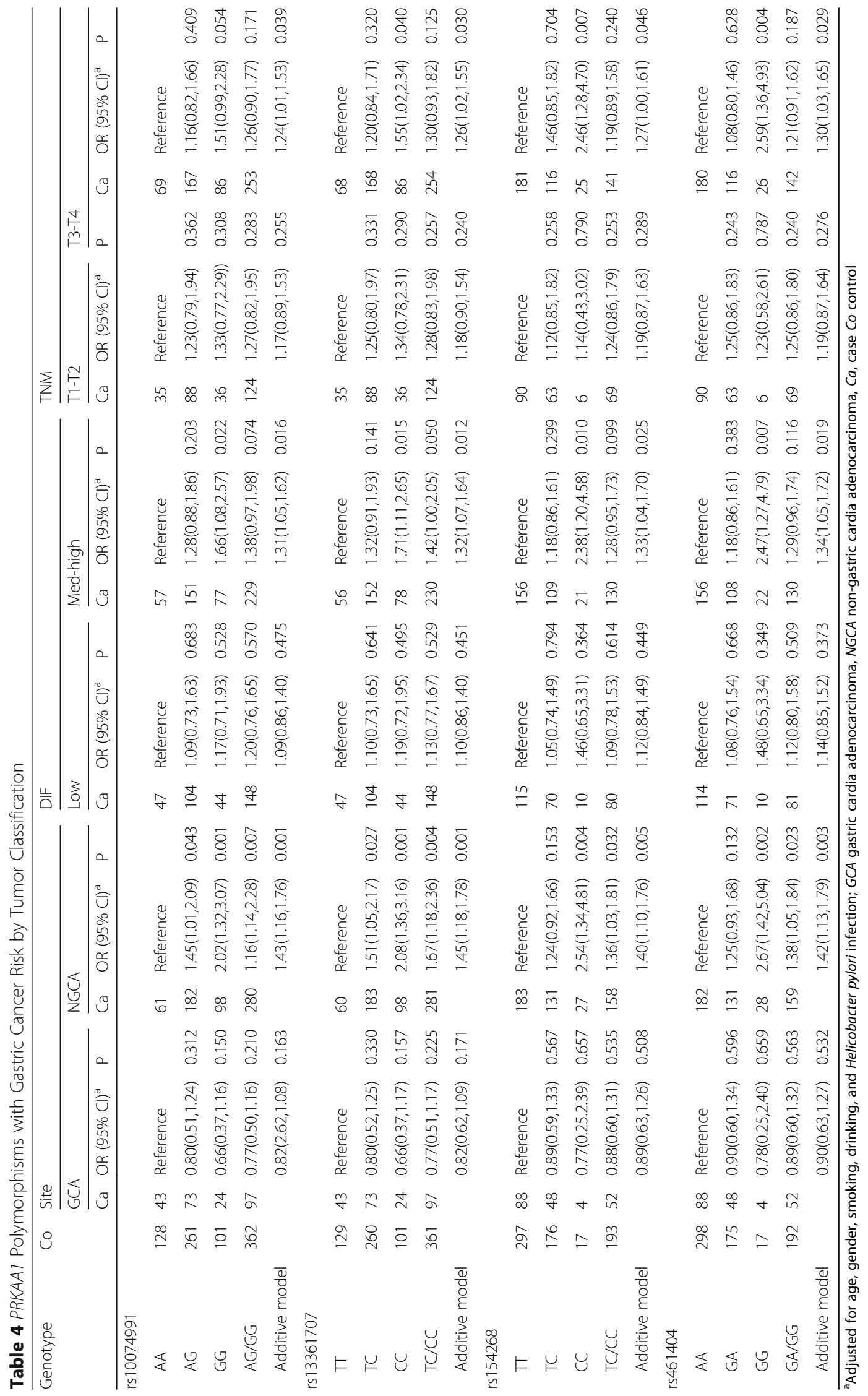


rs10074991, rs13361707, rs3805490, rs154268, rs461404) is associated with the increased risk of $\mathrm{GC}(\mathrm{OR}=1.29$, 95\%CI: $1.02-1.62, p=0.035)$, as compared with other haplotypes (Fig. 1).

\section{Association between polymorphisms and clinical outcome of patients}

A total 481 patients were followed up for the survival state. The association of polymorphisms with the overall survival (OS) of patients was assessed for their predictive value for patients with heterozygous and homozygous genotype, or their combination, compared to the wild genotype. The results revealed that rs10074991 (AG: adjusted $\mathrm{HR}=1.80,95 \% \mathrm{CI}: 1.21-2.67, p=0.004 ; \quad$ GG: adjusted $\mathrm{HR}=1.75,95 \% \mathrm{CI}: 1.13-2.70, p=0.012$; AG/ GG: $\quad \mathrm{HR}=1.78, \quad 95 \% \mathrm{CI}: 1.21-2.61, \quad p=0.003) \quad$ and rs13361707 (TC: adjusted $\mathrm{HR}=1.85,95 \% \mathrm{CI}: 1.24-2.77$, $p=0.003$; CC: adjusted HR $=1.79,95 \%$ CI: $1.16-2.78, p=$ 0.009; TC/CC: adjusted HR $=1.83,95 \%$ CI: $1.24-2.70, p$ $=0.002$ ) were associated with poor OS of patients with NCGC, indicating these two polymorphisms have a significant prediction value for the patients with NCGC, as shown in Table 5.

\section{Discussion}

This study revealed that PRKAA1 genetic polymorphismsrs13361707CC, rs10074991GG, rs461404GG, and rs154268CC were associated with increased risk of GC. The susceptibility of these four polymorphisms to the risk of GC were here observed in the subgroup of age $\leq 60$, male, NCGC, median to high differentiation and T3-T4 subgroup. Polymorphisms rs13361707 and rs10074991 were associated with poor survival of patients with NCGC.

Variant rs13361707 is located in the first intron of PRKAA1 at $5 \mathrm{p} 13.1$, which was primarily found to be associated with NCGC risk by a GWAS in a Chinese population(1006 non-cardia gastric cancer and 2273 controls, and confirmed with 3288 with non-cardia gastric cancer and 3609 controls) [8], and the significant association was duplicated by other studies on Chinese population(1124 cases and 1,194controls) [14] and on Korean population (Kim et al.: 477 case-control pairs; Song et al.: 3245 cases and 1700 controls) $[15,16]$. This study observed that rs13361707 CC genotype was associated with increased risk of $\mathrm{GC}$, and $\mathrm{C}$ allele carriers had a higher risk of NCGC, but not of GCA, indicating the association of rs13361707with the increased GC risk is specific to NCGC. Etiological studies have found differences between GCA and NCGC, concerning e.g. $H$. pylori infection [17, 18], or body mass index [19], and which was confirmed by epidemiological study that also suggested the susceptibility of genetic polymorphism to GC is different for NCGC and GCA [20]. Moreover, in the subgroup of positive $H$. pylori infection, our study showed rs13361707CC genotype is associated with increased risk of $\mathrm{GC}$, indicating the interaction of rs13361707 and H. pylori can enhance the GC risk, which is consistent with the results of previous study [21]. The polymorphism rs13361707 is located in the first intron of PRKAA1 gene, which is a cellular energy sensor maintaining energy homeostasis, and contributes to cancer development by regulating mRNA translation and protein synthesis $[22,23]$. Although the function of rs13361707 is largely unknown, several published studies and the current work indicated that risk of rs13361707 for GC was associated with the type of GC, and its susceptibility may be influenced by H. pylori infection [5].

This study also showed that rs10074991GG genotype is borderline significantly associated with increased risk of $\mathrm{GC}$, and stratification analysis revealed the genotype to be associated with increased risk of NCGC, which is consistent with the reports of $\mathrm{Hu}$ et al. [20] that

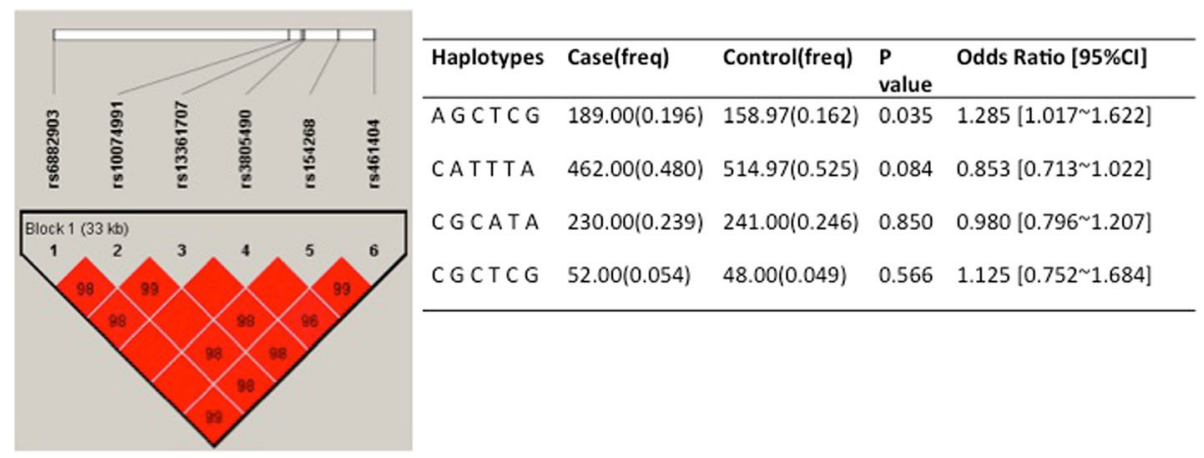

Fig. 1 Haplotype analysis of polymorphisms indicating the susceptibility to gastric cancer risk. The linkage disequilibrium (LD) map according to the genotype data, the color and figure show the linkage disequilibrium coefficient with D' values The prevalence of haplotype A-G-C-T-C-G (rs6882903, rs10074991, rs13361707, rs3805490, rs154268, rs461404) was significantly higher among cases (19.6\%) compared to controls (16.2\%) (haplotype-specific $p=0.035)$, and those with this haplotype have 1.29 times higher risk of gastric cancer $(\mathrm{OR}=1.29,95 \% \mathrm{Cl}: 1.02-1.62, p=0.035)$ compared to noncarriers 
Table 5 PRKAA1 Polymorphisms with clinical outcome of patients with NGCA

\begin{tabular}{|c|c|c|c|c|c|c|c|c|}
\hline \multirow[t]{2}{*}{ Genotype } & \multicolumn{2}{|l|}{ All patients } & \multicolumn{6}{|l|}{ NGCA } \\
\hline & HR (95\% Cl) & $p$-Value & HR (95\% Cl) & $p$-Value & $\operatorname{HR}(95 \% \mathrm{Cl})^{\mathrm{a}}$ & $p$-Value & $\mathrm{HR}(95 \% \mathrm{Cl})^{\mathrm{b}}$ & $p$-Value \\
\hline \multicolumn{9}{|l|}{ rs10074991 } \\
\hline AA & Reference & & Reference & & Reference & & Reference & \\
\hline$A G$ & $1.16(0.87,1.55)$ & 0.300 & $1.62(1.10,2.38)$ & 0.015 & $1.63(1.10,2.41)$ & 0.015 & $1.80(1.21,2.67)$ & 0.004 \\
\hline GG & $1.15(0.83,1.59)$ & 0.401 & $1.64(1.08,2.49)$ & 0.020 & $1.71(1.12,2.60)$ & 0.012 & $1.75(1.13,2.70)$ & 0.012 \\
\hline $\mathrm{AG} / \mathrm{GG}$ & $1.16(0.88,1.52)$ & 0.290 & $1.62(1.12,2.35)$ & 0.011 & $1.66(1.14,2.41)$ & 0.008 & $1.78(1.21,2.61)$ & 0.003 \\
\hline \multicolumn{9}{|l|}{ rs13361707 } \\
\hline$\pi$ & Reference & & Reference & & Reference & & Reference & \\
\hline $\mathrm{TC}$ & $1.18(0.89,1.57)$ & 0.253 & $1.67(1.13,2.47)$ & 0.010 & $1.68(1.13,2.50)$ & 0.010 & $1.85(1.24,2.77)$ & 0.003 \\
\hline CC & $1.16(0.84,1.61)$ & 0.364 & $1.68(1.10,2.56)$ & 0.016 & $1.76(1.15,2.68)$ & 0.009 & $1.79(1.16,2.78)$ & 0.009 \\
\hline $\mathrm{TC} / \mathrm{CC}$ & $1.18(0.90,1.54)$ & 0.246 & $1.67(1.15,2.44)$ & 0.007 & $1.71(1.17,2.50)$ & 0.006 & $1.83(1.24,2.70)$ & 0.002 \\
\hline \multicolumn{9}{|l|}{ rs154268 } \\
\hline$\pi$ & Reference & & Reference & & & & & \\
\hline $\mathrm{TC}$ & $1.08(0.85,1.36)$ & 0.525 & $1.18(0.90,1.56)$ & 0.242 & & & & \\
\hline CC & $1.23(0.78,1.94)$ & 0.367 & $1.29(0.78,2.12)$ & 0.322 & & & & \\
\hline $\mathrm{TC} / \mathrm{CC}$ & $1.10(0.88,1.17)$ & 0.405 & $1.20(0.92,1.56)$ & 0.183 & & & & \\
\hline \multicolumn{9}{|l|}{ rs6882903 } \\
\hline CC & Reference & & Reference & & & & & \\
\hline CA & $0.97(0.76,1.24)$ & 0.796 & $1.12(0.84,1.48)$ & 0.443 & & & & \\
\hline AA & $1.48(0.89,2.47)$ & 0.130 & $1.56(0.90,2.72)$ & 0.113 & & & & \\
\hline CA/AA & $1.02(0.81,1.29)$ & 0.869 & $1.17(0.89,1.53)$ & 0.252 & & & & \\
\hline \multicolumn{9}{|l|}{ rs3805490 } \\
\hline$\pi$ & Reference & & Reference & & & & & \\
\hline TA & $1.03(0.82,1.30)$ & 0.807 & $1.07(0.81,1.41)$ & 0.626 & & & & \\
\hline AA & $0.82(0.51,1.31)$ & 0.404 & $0.97(0.55,1.68)$ & 0.898 & & & & \\
\hline TA/AA & $0.99(0.80,1.24)$ & 0.953 & $1.06(0.81,1.37)$ & 0.691 & & & & \\
\hline \multicolumn{9}{|l|}{ rs461404 } \\
\hline AA & Reference & & Reference & & & & & \\
\hline GA & $1.08(0.86,1.37)$ & 0.506 & $1.19(0.90,1.56)$ & 0.352 & & & & \\
\hline GG & $1.25(0.80,1.94)$ & 0.333 & $1.31(0.80,2.13)$ & 0.228 & & & & \\
\hline GA/GG & $1.11(0.89,1.38)$ & 0.379 & $1.21(0.93,1.57)$ & 0.165 & & & & \\
\hline
\end{tabular}

rs10074991 G allele linked with rs13361707 C allele (these two polymorphisms locate in the intron of PRKAA1 with the distance of $1333 \mathrm{bp)}$ was a risk factor of NCGC. Moreover, such an association was also reported by Kim et al. [15] in a Korean population [15]. However, the function of these two sites remains unclear and the mechanism has yet to be established.

In this study, rs154268 CC genotype was also found to be associated with increased risk of GC for all participants and especially for the subgroup of NCGC, tumor with median to high differentiation, and T3-T4, suggesting rs154268 could be associated with pathological characteristics of GC. Consistent with this, the rs154268 TC genotype was also previously reported to be associated with the risk of GC [15], indicating that the C allele is a risk factor for GC. Actually, this study revealed the linkage disequilibrium (LD) between rs154268 and rs461404 $\left(D^{\prime}=1.0\right)$, which means the result of rs461404 is in accord with that of rs154268. However, to date, there is no functional study regarding the potential functional role of these two polymorphisms in carcinogenesis. In general, in this study, the result of rs461404 was inconsistent with that of rs154268.

The present work showed that rs10074991 G and rs13361707 C allele carriers with NCGC have poor OS, and this association was still observed after being adjusted by basic clinical characteristics (age, gender, $H$. pylori infection, drinking, and smoking) or pathological 
characteristics (tumor differentiation, tumor stage), indicating these two polymorphisms were independent factors for predicting the clinical outcome for NCGC. To our knowledge, this is the first report to discuss the role of these two polymorphisms in prognosis for patients with NCGC, which however should be verified by a further research with larger samples.

There are some limitations of this study. First, the sample size is relatively small, which may limit the statistical power, especially for the multiple stratified analyses. Second, the polymorphisms discussed in this study were limited in number and based on previous knowledge of potential functional significance of polymorphisms that have been found to be related to GC risk. Thus, a more comprehensive tagging SNP-based approach and a haplotype block analysis would better assesses the association and provides more complete information regarding the associations of AMPK pathway genes and GC risk.

\section{Conclusions}

This case-control study provided the evidence that rs13361707CC, rs10074991GG, rs461404GG, and rs154268CC are associated with increased GC risk, especially for NCGC, and that rs10074991 G and rs13361707 $\mathrm{C}$ alleles are independent prognostic factors for NCGC.

\section{Additional file}

Additional file 1: Polymorphism position and minor allele frequency. (DOC $35 \mathrm{~kb}$ )

\section{Abbreviations}

AMPK: 5'-AMP-activated protein kinase; Cl: confidence intervals; GCA: gastric cardiac adenocarcinoma; GWAS: genome-wide association study; $H$. pylori: Helicobacter pylori; HR: hazard ration; HWE: Hardy-Weinberg Equilibrium; NCGC: non-cardiac gastric cancer; OR: odds ratios; OS: overall survival

\section{Acknowledgements}

This work is supported by the National Natural Science Foundation (Grant numbers: 81472786, 81472305, 81773192); Suzhou Municipal Health Bureau projects (Grant number: LCZX201318); The Foundation of tumor clinical and basic research team (KYC005); The Six Talents Peak Project of Jiangsu Province (2014-WSW-061)

\section{Availability of data and materials}

Data supporting our findings are presented in the "Results" section. Researchers interested in source data are invited to write to the corresponding author.

\section{Authors' contributions}

MC, BJ and PL designed the study. B.H collected the sample and information. M.T and PW performed the statistical analysis and drafted and revised the manuscript. LC and JL participated with the $H$. pylori detection and data collection. All authors read and approved the final version of the manuscript.

\section{Ethics approval and consent to participate}

The protocol of this study was in accordance with the Declaration of Helsinkiand approved by the Institutional Review Board of the Nanjing First Hospital, and written informed consents were obtained from all participants.
Consent for publication

Not applicable.

\section{Competing interests}

The authors declare that they have no competing interests.

\section{Publisher's Note}

Springer Nature remains neutral with regard to jurisdictional claims in published maps and institutional affiliations.

\section{Author details}

${ }^{1}$ Department of Radiotherapy \& Oncology, Kunshan First People's Hospital Affiliated to Jiangsu University, Kunshan, Jiangsu Province, China.

${ }^{2}$ Department of Critical Care Medicine, The affiliated Yixing Hospital of Jiangsu University, Yixing 214200, Jiangsu Province, China. ${ }^{3}$ General Clinical Research center, Nanjing First Hospital, Nanjing Medical University, Nanjing 220006, Jiangsu Province, China. ${ }^{4}$ Departments of Medical biology, Wannan Medical College, Wuhu, Anhui Province, China. ${ }^{5}$ Department of

Gastroenterology, Xuzhou Hospital of Traditional Chinese Medicine Affiliated to Nanjing University of Chinese Medicine, Xuzhou, China. ${ }^{6}$ Departments of Medical Oncology, Jiangsu Cancer Hospital Affiliated to Nanjing Medical University, Jiangsu Province Institute of Cancer, Nanjing, Jiangsu Province, China. ${ }^{7}$ Department of Medical Oncology, Wuxi People's Hospital of Nanjing Medical University, No. 299, Qingyang Road, Wuxi 214023, Jiangsu Province, China

Received: 8 September 2017 Accepted: 13 September 2018

Published online: 25 September 2018

\section{References}

1. Torre LA, Bray F, Siegel RL, Ferlay J, Lortet-Tieulent J, Jemal A. Global cancer statistics, 2012. CA Cancer J Clin. 2015;65(2):87-108.

2. Ang TL, Fock KM. Clinical epidemiology of gastric cancer. Singap Med J. 2014;55(12):621-8

3. Karimi P, Islami F, Anandasabapathy S, Freedman ND, Kamangar F. Gastric cancer: descriptive epidemiology, risk factors, screening, and prevention. Cancer Epidemiol Biomarkers Prev. 2014;23(5):700-13.

4. Hemminki K, Sundquist J, Ji J. Familial risk for gastric carcinoma: an updated study from Sweden. Br J Cancer. 2007;96(8):1272-7.

5. Mocellin S, Verdi D, Pooley KA, Nitti D. Genetic variation and gastric cancer risk: a field synopsis and meta-analysis. Gut. 2015;64(8):1209-19.

6. Piao Y, Li Y, Xu Q, Liu JW, Xing CZ, Xie XD, Yuan Y. Association of MTOR and AKT gene polymorphisms with susceptibility and survival of gastric Cancer. PLoS One. 2015;10(8):e0136447

7. Hyland $\mathrm{PL}$, Lin SW, Hu N, Zhang H, Wang L, Su H, Wang C, Ding T, Tang ZZ, Fan $J$, et al. Genetic variants in fas signaling pathway genes and risk of gastric cancer. Int J Cancer. 2014;134(4):822-31.

8. Shi Y, Hu Z, Wu C, Dai J, Li H, Dong J, Wang M, Miao X, Zhou Y, Lu F, et al. A genome-wide association study identifies new susceptibility loci for noncardia gastric cancer at 3q13.31 and 5p13.1. Nat Genet. 2011;43(12):1215-8.

9. Dong Y, Chen J, Chen Z, Tian C, Lu H, Ruan J, Yang W. Evaluating the Association of Eight Polymorphisms with Cancer susceptibility in a Han Chinese population. PLoS One. 2015;10(7):e0132797.

10. He B, Pan Y, Xu Y, Deng Q, Sun H, Gao T, Wang S. Associations of polymorphisms in microRNAs with female breast cancer risk in Chinese population. Tumour Biol. 2015;36(6):4575-82.

11. He BS, Pan YQ, Lin K, Ying HQ, Wang F, Deng QW, Sun HL, Gao TY, Wang SK. Evaluation the susceptibility of five polymorphisms in microRNA-binding sites to female breast cancer risk in Chinese population. Gene. 2015;573(1):160-5.

12. Gabriel S, Ziaugra L, Tabbaa D: SNP genotyping using the Sequenom MassARRAY iPLEX platform. Curr Protoc Hum Genet. 2009; Chapter 2:Unit 212.

13. Thomas RK, Baker AC, Debiasi RM, Winckler W, Laframboise T, Lin WM, Wang M, Feng W, Zander T, MacConaill L, et al. High-throughput oncogene mutation profiling in human cancer. Nat Genet. 2007;39(3):347-51.

14. Qiu LX, He J, Cheng L, Zhou F, Wang MY, Sun MH, Zhou XY, Li J, Guo WJ, Wang YN, et al. Genetic variant of PRKAA1 and gastric cancer risk in an eastern Chinese population. Oncotarget. 2015:6(40):42661-6.

15. Kim YD, Yim DH, Eom SY, Moon SI, Yun HY, Song YJ, Youn SJ, Hyun T, Park JS, Kim BS, et al. Risk of gastric cancer is associated with PRKAA1 gene polymorphisms in Koreans. World J Gastroenterol. 2014;20(26):8592-8. 
16. Song HR, Kim HN, Kweon SS, Choi JS, Shim HJ, Cho SH, Chung IJ, Park YK, Kim SH, Choi YD, et al. Genetic variations in the PRKAA1 and ZBTB20 genes and gastric cancer susceptibility in a Korean population. Mol Carcinog. 2013; 52(Suppl 1):E155-60.

17. Kamangar F, Dawsey SM, Blaser MJ, Perez-Perez GI, Pietinen P, Newschaffer CJ, Abnet CC, Albanes D, Virtamo J, Taylor PR. Opposing risks of gastric cardia and noncardia gastric adenocarcinomas associated with helicobacter pylori seropositivity. J Natl Cancer Inst. 2006;98(20):1445-52.

18. Kamangar F, Qiao YL, Blaser MJ, Sun XD, Katki H, Fan JH, Perez-Perez Gl, Abnet CC, Zhao P, Mark SD, et al. Helicobacter pylori and oesophageal and gastric cancers in a prospective study in China. Br J Cancer. 2007;96(1):172-6.

19. Abnet CC, Freedman ND, Hollenbeck AR, Fraumeni JF, Jr., Leitzmann M, Schatzkin A: A prospective study of BMl and risk of oesophageal and gastric adenocarcinoma. Eur J Cancer 2008, 44(3):465-471.

20. Hu N, Wang Z, Song X, Wei L, Kim BS, Freedman ND, Baek J, Burdette L, Chang J, Chung C, et al. Genome-wide association study of gastric adenocarcinoma in Asia: a comparison of associations between cardia and non-cardia tumours. Gut. 2015.

21. Cai M, Dai S, Chen W, Xia C, Lu L, Dai S, Qi J, Wang M, Wang M, Zhou L, et al. Environmental factors, seven GWAS-identified susceptibility loci, and risk of gastric cancer and its precursors in a Chinese population. Cancer Med. 2017:6(3):708-20.

22. Krishan S, Richardson DR, Sahni S. Gene of the month. AMP kinase (PRKAA1). J Clin Pathol. 2014;67(9):758-63.

23. van Veelen W, Korsse SE, van de Laar L, Peppelenbosch MP. The long and winding road to rational treatment of cancer associated with LKB1/AMPK TSC/mTORC1 signaling. Oncogene. 2011;30(20):2289-303.

Ready to submit your research? Choose BMC and benefit from:

- fast, convenient online submission

- thorough peer review by experienced researchers in your field

- rapid publication on acceptance

- support for research data, including large and complex data types

- gold Open Access which fosters wider collaboration and increased citations

- maximum visibility for your research: over $100 \mathrm{M}$ website views per year

At $\mathrm{BMC}$, research is always in progress.

Learn more biomedcentral.com/submissions 